\title{
Conquest Pro 12 coronary guidewire for perforation of functionally interrupted aorta
}

\author{
Keywords: Interventional catheterisation; coarctation; stent; children; guidewire \\ Received: 2 July 2012; Accepted: 2 October 2012; First published online: 12 November 2012
}

We read an article by Alwi et al $^{1}$ entitled "Pulmonary atresia with intact septum: the use of Conquest Pro coronary guidewire for perforation of atretic valve and subsequent interventions" with deep interest. It is important to mention that guidewires used in chronic total occlusion treatment such as Conquest Pro are often used by adult cardiologists, but are not used in paediatric cardiology because of not being known or because of limited areas of usage. The guidewires used in chronic total occlusion differ from other guidewires in that they have heavy tips. They are placed into an exact area where they are desired to be perforated and then they are pushed gently, which is sufficient to have successful results. In the following extract, we would like to share our experience with Conquest Pro 12, which we used successfully during transcatheter operation in a patient detected with functionally interrupted aorta.

A 21-year-old female patient was referred to our hospital as she had been diagnosed with interrupted aorta while having been tested for hypertension. A computer tomography scan detected a $3-\mathrm{mm}$ membranous-type interruption at $1 \mathrm{~cm}$ distal to the left subclavian artery, and thus transcatheter intervention was scheduled (Fig 1). The various guidewires that were sent from the sheath placed in the left radial artery could not pass the atretic portion, despite the sharp edges of these guidewires. Radiofrequency perforation (HAT 300 generator and 0.018-in. cereblate wire (Sulzer Osypka GmBH, Grenzach-Wyhlen, Germany)) was tried by giving energy up to $20 \mathrm{~W}$ in order to perforate the atretic portion; however, this was not achieved.

Correspondence to: Dr I. C. Tanidir, MD, Department of Pediatric Cardiology, İstanbul Mehmet Akif Ersoy, Thoracic and Cardiovascular Surgery Center and Research Hospital, Istanbul, Turkey. Tel: +90 21269220 00; Fax: +90 212 47194 94; E-mail: cansaran@yahoo.com
On the contrary, a positive result was obtained when the Conquest Pro 12 (Asahi Intecc Co. Ltd, Aichi, Japan) guidewire perforation procedure was performed. During the first trial, the guidewire was pulled back when it was realised that the wire had advanced subintimally, and only after repeating the procedure did it enter the exact lumen. A loop was formed between the left radial artery and the left femoral artery. Once pre-dilatation had been done, first by $4 \mathrm{~mm} \times 2 \mathrm{~cm}$ coronary balloon and later by $7 \mathrm{~mm} \times 2 \mathrm{~cm}$ Tyshak Balloon (Numed Canada Incorporation, Cornwall, Ontario, Canada) and lumen was generated, $39 \mathrm{~mm}$ coated $\mathrm{CP}$ stent was placed there with standard technique. Continuity in the aorta was provided as the atretic part was opened (Figs 1 and 2).

In the case described above, unlike Alwi et al, after radiofrequency perforation was found to be unsuccessful, the Conquest Pro guidewire was used. Despite the fact that perforation did not occur with the help of the radiofrequency catheter, successful perforation was achieved with the use of the Conquest Pro 12 guidewire. The Conquest Pro 12 guidewire is heavier when compared with radiofrequency perforation and possesses a higher penetration power. As in the case described above, subintimal advancement occurred despite the fact that the guidewire was being used in an adult patient; on the contrary, it is thought that this wire has a high risk of misperforation in newborn patients. In order to avoid this, as Alwi et $\mathrm{al}^{1}$ have emphasised, total placement of the guidewire into the lesion side and its use for straightforward cases are very crucial points. In addition, it is very important to manipulate the guidewires gently. Owing to the fact that the edge of the guidewire is much heavier than normal, it is accompanied by severe perforation risk. In the adult patients diagnosed with chronic total occlusion, perforation and tamponade rates during the use of Conquest Pro 

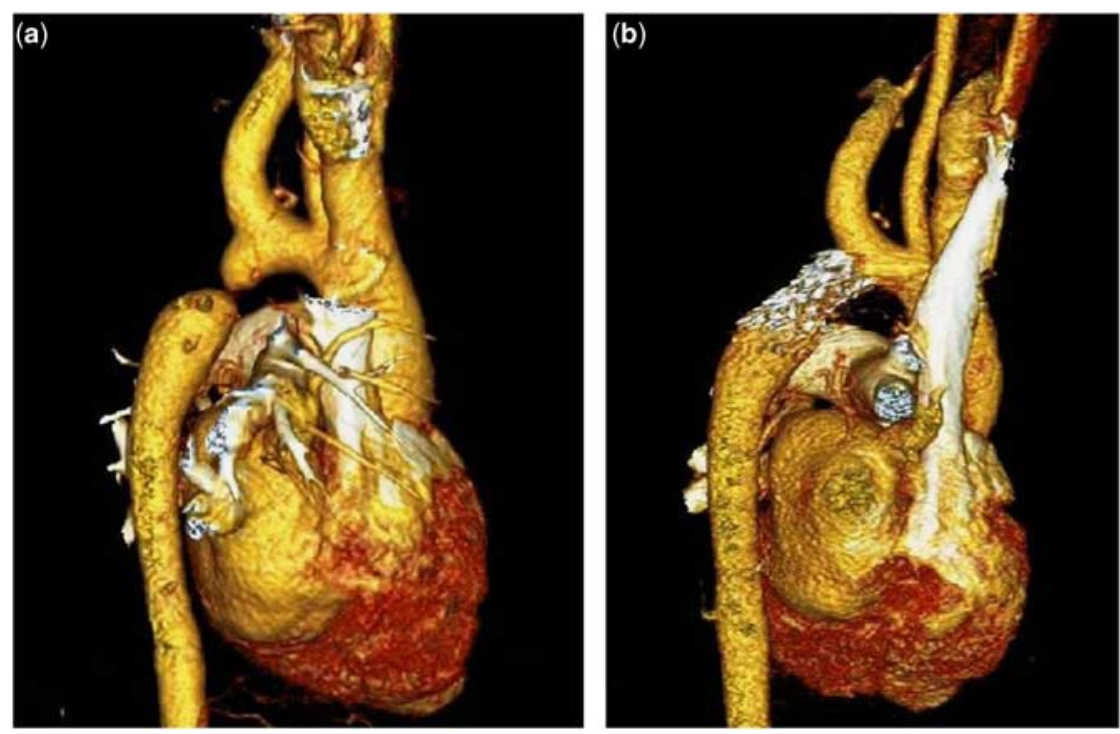

Figure 1.

Three-dimensional computed tomography images of the patient before $(\boldsymbol{a})$ and after $(\boldsymbol{b})$ the procedure.

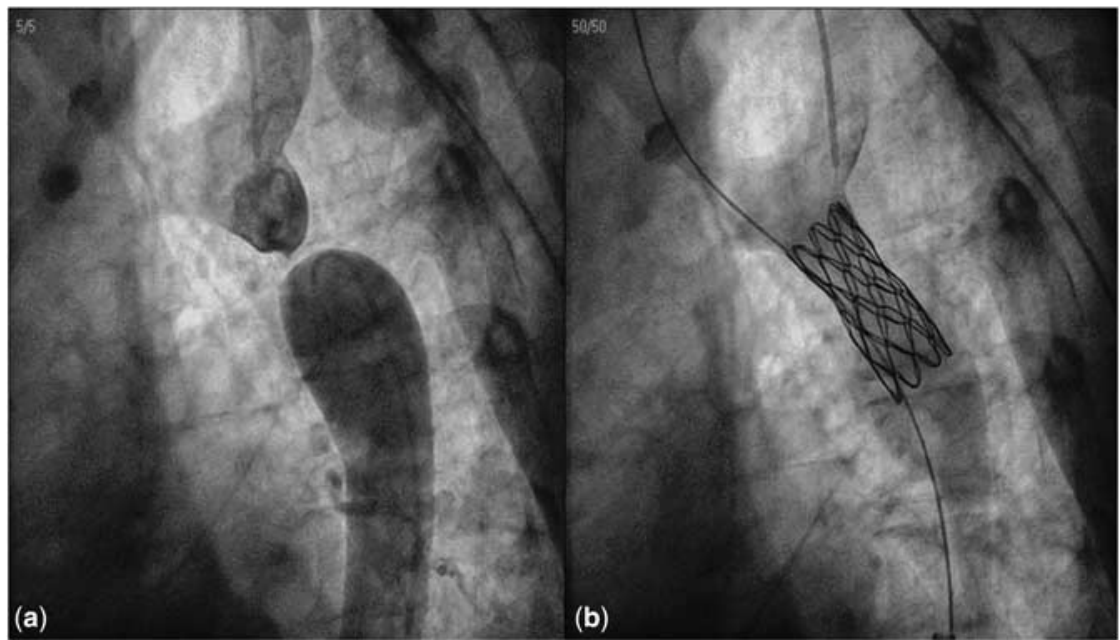

Figure 2.

Lateral descending aortagrams of the patient before $(\boldsymbol{a})$ and after $(\boldsymbol{b})$ the procedure.

were reported as $7.8 \%$ and $1.7 \%$, respectively. ${ }^{2}$ Another technical detail we can mention according to our chronic total occlusion experience is that curving the edge of the Conquest Pro guidewire in special cases by bending the tip of the catheter would be beneficial.

In conclusion, for perforation of the membranous atretic tissues, either as the first choice or when radiofrequency perforation is found to be unsuccessful, Conquest Pro or the catheters used in chronic total occlusion treatment such as Conquest Pro 12 can be successfully used. Owing to the fact that perforation risk is high even in adult patients, it should be used cautiously in children, ensuring that coated stents and a surgery team is kept ready should complications arise.

\section{Ender Odemis, Ibrabim C. Tanidir, Alper Guzeltas Department of Pediatric Cardiology}

Nevzat Uslu

Department of Cardiology, Istanbul Mebmet Akif Ersoy, Thoracic and Cardiovascular Surgery Center and Research Hospital, Istanbul, Turkey 
Vol. 23, No. 4

\section{References}

1. Alwi M, Budi RR, Mood MC, Leong MC, Samion H. Pulmonary atresia with intact septum: the use of Conquest Pro coronary guidewire for perforation of atretic valve and subsequent interventions. Cardiol Young 2012; 29: 1-6.

2. Mitsudo K, Yamashita T, Asakura Y, et al. Recanalization strategy for chronic total occlusions with tapered and stiff-tip guidewire. The results of CTO new techniQUE for STandard procedure (CONQUEST) trial. J Invasive Cardiol 2008; 20: 571-577. 